\title{
Mikroskopische Untersuchungen mancher Gesteins-Proben von Böhrek-Massiv
}

\author{
von \\ N. ILGÜZ \\ (Geologische Institut der Universität Ankara)
}

Öxet: 1951 yılında Keskinin güney batısındaki Böhrek dağından ve De: nek day̆ının bir kısmından muhtelif taş nümuneleri alınmış ve bunlardan Böhrek dağıa ait nümuneler mikroskopla incelenmiştir. Bu araştırmalarla Böhrek masifinin esas itibariyle Hornblende-Granitlerdeo, ara sira rastlanan Damar-Kayalardan teşekkül ettił̆ neticesine varılmıştır.

Böhrek, Denek ve Kırşehir massiflerinin yaşları hakkında muhtelif araştırıcılar tarafından ģeşitli fikirler ortaya atılmış bulunmaktadır. Yaptığımız müşahedeler, araştırma bölgesinde vukubulan entruzyonların Mezozoik sonlarına doğru başlamış olduğu kanaatıaı uyandırmıştır.

Esas mineralleri Feldspat (Ortoklaz, Oligoklaz, Andezin), kuvars, Hornblende ve az nikdarda Biotit ve Ojitlerden ibaret olan bu massifin diger minerallerini Magnetit, Apatit ve Stenler teşkil ederler. Bütün uümunelerde Feldspatlar taşın hâkim bir mineral ceşidi olarak kalmakta, yalnız bazan plajioklazlar Ortoklazlardan daha yüksek bir mikdarda* mineralojik bile* şime iştirak etmektedirler. Buna karşılık diğer esas mineraller daimâ dẹ̆işik mikdarlar gösterirler. Bu suretle massif, kuvarsdiorite dogru giden bir bileşim arzeder. Hattâ massifin kuzey doğu kenar kısımları́da Alaskitlere de rastlanmıştır. Mineralojik bileşimlerde görülen bu deģişiklikler bazan yapılarda da görülür. Bir q̧ok nümuaeler Hypidiomorph-taneli bir yapıya mâlik oldukları halde bazlları Porphyroid bir yapı göstermektedirler.

Aplit, Ojitli Siyenit-Aplit ve Ojit-Kersantit olduklar tesbit edilen kayalar aynı Mağmantn sonradan vukubulan enjeksison mahsulları olsalar gerektir.*

Waehrend einer Reise im Jahre 1951 habe ich am BöhrekDağ südwestlich von Keskin und an einem Teil des Deneks von Keskin, eine Reihe von Gesteinsproben gesammelt und die Probestücke von Böhrek mikroskopisch untersucht. Die Ergebnisse haben gezeigt, dass das Massiv aus den intrusiven Gesteinen und zwar haupsaechlich aus Hornblende-Graniten aufgebaut worden ist. Hier und da sind vertreter der Ganggesteine

* Bu yazımla mineralojik bileşimlerini açıklamaya çalıştığım nümunelerin ince kesitleri Ístanbul Teknik üniversitesince yaptırılmış oldugundan burada jeoloji Enstitüsü Müdürü Sayıu Prof. Malik Sayar'la Sayın Doçent Kemal Erguvanh'ya teşekkür ederim. 
auch vorhanden. Sie scheinen aber die nachtraeglichen Durchbrüche und Injektionen desselben Magmas zu sein.

Über das Alter des Böhrek-, Denek-u. Kırşehir-Massivs sind die Meinungen verschieden. Nach E. Chaput ${ }^{2)}$ soll es ein altes Massiv sein. P. Arni ${ }^{3)}$, schreibt dem Massiv ein vortertiaeres Alter zu. Nach Dr. A. Maucher'), am Denek könnten die nüm: mülitische Kalke durch die granitische Intrusionen metamorphosiert worden sein "weil er den Aufschluss nicht selbst gesehen hat". Nach F. Baykal ${ }^{5}$ ) und Ch. Ladame ${ }^{6)}$, soll das Massiv dagegen im mesozoischen Zeit-Raum gebildet sein. V. Koven$\mathrm{ko}^{7)}$ aber uimmt an, dass den grösstenteil von Intrusionen am Denek nach der jurassischen Zeit stattgefunden haben.

Am Böhrek-Dağ selbst im Untersuchungsgebiete habe ich keinen guten Aufschluss beobachtet. Aber am Hange des Cabatözü-Tals und beim Dorfe Ceritkale, südlich vom Denek, sind solche Aufschlüsse vorhanden. An diesen liegen die alttertiaeren konglomeratischen Sandsteine "Arkos" und Basal-Konglomerate über dem Granit. Diese Sandsteine und Konglomerate liegen unmittelbar in der Naehe vom Dorfe Haciali-Obası und Ceritkale unter dem nümmülitischen Kalke "Lütetien". Die erwaehnten Konglomerate, welche von den Granitgeröllen gebildet sind, und Sandsteine zeigen aber fast keinen Spur von Metamorphismus. Daher muss dieses Massif wie P. Arni ${ }^{3)}$ auch behauptete, einen vortertiaren Zeitalter haben. In Keskin und ihrer naeheren Umgebung überlagern die Marmore granitischen Gesteine. Hier unmittelbar südlich von Keskin an der Kontaktstelle stellte ich einige Kontaktmineralien "Granat, Hematit" und unter den Marmoren metamorphosierte Sandstein reste fest. Danach scheinen der Beginn der Intrusionen hier gegen Ende der mesozoischen Zeit stattgefunden zu haben.

P. de Tchihatcheff ${ }^{1}$ ), schreibt den intrusiven Gesteine von Denek-Böhrek-u. - Çelebidağ im grossen und ganzen ein Syenit zu. Nach E. Chaput ${ }^{2)}$, besteht das Massiv des Böhreks aus den Amphibolhaltigen Graniten und Quarzdioriten. Nach M. Ch. Ladame ${ }^{6)}$, die aus der Umgebung der Molybden Lagersraetten des Deneks gesammelten Granitprobestücke sind Biotit-Hornblende-u. -quarzhaltige Gesteine, welche hie und da zu den quarzhaltigen Dioriten übergehen. Nach Dr. A. Maucher", bestehen diese Granite aus Ortoklas, Plagioklas "Oligoklas, Andesin", Quarz, Biotit und akzessorisch aus Apatit Nach V. Kovenko") aber, 
bildet sich das Denek-Massiv aus Granit, Monzonit und Quarzdiorit.

Die von mir gesammelten Probestücke von Böhrek.Massiv bestehen aus Feldspat (Ortoklas, Oligoklas, Andesin), Quarz, Hornblende, spaerlich Augit, Biotit und akzessorisch aus Mag. netit, Apatit und Sphen.

Das Massiv baut sich hauptsaechlich aus Kalkalkali-Hornblende.Graniten auf. In diesen Graniten sind die Feldspate immer vorherrschend. Dagegen treten die andere Hauptgemengteilen in wechselnden Mengen zurück. Es kommt vor, dass in manchen Graniten die Plagioklase, in anderen dagegen die Ortoklase vorherrschen, so dass das Massiv nach Quarzdiorit übergeht. Es treten auch am nordöstlichen Rande des Massivs Alaskite auf. An manchen Stellen bemerkt man sogar die Strukturaenderungen. Viele Stücke zeigen naemlich eine hypidiomorphkörnige Struktur, die übrigen sind dagegen porphyroid.

Die mikroskopische Untersuchungsergebnisse dieser Probestücke sind unten angegeben :

\section{G R A N I T E}

No. 116/43 Hornblende Granit

Das Gestein ist körnig und hat rötlich graue Farbe. Makroskopisch sind körner von Feldspat, Quarz und kleine Kristaellchen von Hornblende erkennbar.

U. d. Mikroskop stellte ich eine Menge von Feldspat, Quarz, und spaerlich Augit und Biotit fest. Ausserdem treten akzessorisch Sphen, Apatit und Magnetit auf.

Die Ortoklase kommen im Dünnschliff reichlicher als die Plagioklase vor. Sie sind meist verzwillingt und zum Teil kaolinisiert und auch sericitisiert. Die Ortoklas-Kristaellchen sind oft grösser als die anderen Mineralien. Sie haben Einschlüsse von Hornblende, Magnetit, Biotit, Apatit, und Sphen.

Die Plağioklase sind durch die Oligoklase und mit $35 \% \mathrm{An}$. haltigen Andesine vertreten. Diese letzteren kommen viel weniger als die ersteren vor. Manche Plagioklase treten in polysynthetisch verzwillingten Formen vor. Die Auslöschung mancher Stücke ist ondulös. Ich stellte in Plagioklasen als Einschlüsse von Hornblende, Apatit und einen Augit fest. 
Die Gewöhnliche Hornblenden bilden den zweite. Hauptgmengteil des Gesteins. Sie treten im Dünnschliff als Laengs-u. Querschnitten auf. Die Auslöschungsschiefe der Hornblenden betraegt $24^{\circ}$. Sie zeigen folgenden Pleochroismns :

$$
\begin{aligned}
& n_{g}=\text { Dunkel grün } \\
& n_{m}=\text { Grünlich" leicht gelb. } \\
& n_{p}=\text { Grient }
\end{aligned}
$$

Den dritten Gemengteil des Gesteins bilden die Körner von Quarz. Sie treten im Vergleich zu Hornblenden etwas zurück. Auch in diesem sind viele Einschlüsse von Hornblende, Plagioklas, Apatit und Magnetit. Die meisten von ihnen zeichnen sich durch ondulöse Auslöschungen aus.

\section{No. 118/45 Hornblende-Granit}

Das Probestück besteht aus Quarz, Feldspat und einem Gemengteil dunkler Mineralien, die in reichen Mengen im Gestein vertreten sind.

U. d. Mikroskop sieht man ein Hypidiomorphkörniges Gefüge. Die Hauptbestandteile des Gesteins bestehen wie in der vorigen Probe aus Feldspat, Quarz und Hornblende. Augit fehlt in diesem. Aber die Eieschlüsse von Sphen sind noch reichlicher vorhanden.

Die Ortoklase sind vorherrschend und grösser als die Plagioklase. Ihre Grösse erreicht etwa $4,6 \times 2,8 \mathrm{~mm}$. Sie kommen in Karlsbader Zwillingsformen vor, und sind teilweise kaolinisiert, und haben die Einschlüsse von Plagioklas, Hornblende, Magnetit, Sphen, Apatit, und Biotit.

Die Plagioklase sind zum Teil verwittert und dadurch Kaolin und etwas Sericit entstanden. Viele Plagioklase haben einen zonaren Bau mit basischerem Kern. Sie sind Polysnthelisch verzwillingt. Ihre Grösse erreicht $2,3 \times 1,9 \mathrm{~mm}$. Sie sind durch die Oligok lase und mit 40\% An. haltigen Andesine vertreten. Ich stellte im Plagioklase folgende Einschlüsse fest: Biotite, Magnetite, Hornblenden, Sphene, Apatite. Manche Plagioklase haben eine ondulöse Auslöschung.

Die Quarze bilden den zweiten Hauptbestandteil des Gesteins. Ihre Auslöschungen sind ondulös. Inden Quarzkörnern beobachtete ich Einschlüsse von Sphen, Feldspat und Apatit.

Die Hornblenden sind stark pleochroitisch (leicht gelblich 
grửn-dunkel grün) und haben eine Auslöschungsschiefe von $24^{\circ}$.

No. $122 / 49$

Das Probestück besteht fast aus denselben Mineralien wie oben. In diesem fehlt Augit, und die Hornblenden treten etwas zurück.

No. $129 / 56$

Das Handstück hat eine Mineralogische Zusammensetzung wie oben. Nur fehlt in diesem auch Biotit.

No. $123 / 50$

Das Stück hat sich von denselben Mineralien wie das Pro. bestück No. 129/56 gebildet. In diesem sind nur Ortoklas und Quarz reichlicher vorhanden,

$124 \mathrm{a} / 51 \mathrm{a}$

Dasselbe Gestein wie No. $123 / 50$ oben. Nur Ortoklase treten etwas zurück.

No. $120 / 47$

Das Gestein hat ein körniges Gefüge und besteht aus Quarz Feldspat und dunklen Mineralien.

U. d. Mikroskop sieht man, dass das Gestein aus Feldspat, Quarz, Hornblende und spaerlich Augit und Biotit besteht. Im Dünnschliff treten akzessorisch auch Sphen, Magnetit, und Apatit auf.

Die Ortoklase und Plagioklase liegen im Dünnschliff etwa in gleichen Menge vor. Die ersteren sind zum Teil kaolinisiert und sind nach karlsbadem Gesetz verzwillingt. Ihre Grösse erreicht $8,9 \times 2,5 \mathrm{~mm}$.

Die Plagioklase (Oligoklase u.40\% An. haltige Andesine) treten im Dünnschliff în polysynthetischer Zwillingen vor. Sie sind meist zonar gebaut, und kaolinisiert. Die Feldspate sind hier auch Einschlussreich.

In dieser Probe stellte ich ferner einige Biotitblaettchen fest, die zum Teil chloritisiert sind.

Ausser diesen oben beschriebenen Proben habe ich noch andere 5 Proben untersucht, die den verschiedenen Stellen des. erwaehntenMassivs entnommen waren. Aus diesen Untersuchungen ergab es sich, dass diese "5" Proben fast dieselbe minera- 
logische Zusammensetzung und petrographische Beschaffenheit wie oben erwaehnten Gesteinsstücke haben Nur bei den "3" Proben, von denen zwei Stücke porphyroid sind, sind die Plagioklase vorherschend.

\section{No 104/31 Alaskit}

Ein stark verwittertes Gestein mit einem rötlichen Farbton. Am Handstück treten die Quarze deutlich hervor. Die dunklen Mineralien sind dagegen sehr spaerlich und kommen in kleineren Kristaellchen vor.

U.d. Mikroskop sah ich, dass das Gestein aus Feldspat, Quarz und Biotit besteht. Die Feldspate sind stark kaolinisiert. Die Biotite sind sehr spaerlich vertreten.

Das Gestein ist Hypidiomorph-körnig. Die Ortoklase übertreffen der Menge nach die andere Gemengteile. Sie sind meist verzwillingt. Ausser dem Ortoklas treten auch Oligoklase auf.

Die Quarze sind reich überal im Dünnschliff zu sehen. Der Dünnschliff ist von feinen Limonit-Aederchen durchgezogen und und stellenweise braun gefaerbt.

No. $103 / 30$ Alaskit

Das Gestein ist klein-körnig und weisslich grau. Makroskopisch stellte ich im'Probestück Quarz und Feldspat fest. Zu diesen beteiligen sich auch manche dunkle Mineralien, die stark wittert sind.

U. d. Mikroskop beobachtete ich eine körnige Struktur. Manche Quarz-Kristalle erreichen die Grösse von $1,6 \times 1,2 \mathrm{~mm}$. Die Feldspate sind im allgemeinen stark verwittert und getrübt wobei sind wohl Kaoline entsdanden.

Die Ortoklase treten im Dünnschliff etwas mehr als die Plagioklase vor. Die Ortoklase haben die Einschlüsse von Plagioklase.

Die Plagioklase sind oft polysynthetisch verzwillingt und durch die Oligoklase vertreten. Ihr An. Gehalt $20 \%$.

Die Quazze sind reichlich vorhanden. In ihnen beobachtete ich einige Einschlüsse von Muscovit Blaettchen. Ferner sind im Dünnschliff einige Biotit-Blaettchen mit spaerlich vertretenem Magnetit nachgewiesen worden. 


\section{GA N G G E S TEIN E}

No. $119 / 46$ Aplit

Das Handstück ist feinkörnig und rötlich gefaerbt.

U. d. Mikroskop stellte ich ein körniges Gefüge fest. Die Hauptgemengteile des Gesteins sind Feldspat (Ortoklas, Plagioklas) und Quarz.

Die Plagioklase sind durch (20\% An.-Gehalt) die Oligoklase vertreten. Sie sind polysynthetisch verzwillingt und verwittert und haben einige Einschlïsse von Biotit-Blaettchen. Auch die Ortoklase sind viel staerker verwittert und hahen die Einschlüsse von Biotit und Muscovit.

Die Quarze kommen im Dünnschliff reichlich vor, und haben Einschlüsse von Feldspat und Biotit. An manche Stellen sind Biotite chloritisiert.

No. $123 / 50 / 1$ Aplit

Das Gestein ist feinkörnig und hat eine helle Farbe. Makroskopisch sind Quarz und Feldspat erkennbar. Die dunklen Mineralien treten sehr zurück.

U. d. Mikroskop stellte ich Quarz und Feldspat fest. Die letzteren sind vorherrschend. Unter den Feldspaten kommen die Ortoklase in sehr reichlichen Mengen vor. Die Plagioklase sind dagegen spaerlich vertreten. Sie sind zum Teil verwittert und dadurch Kaolin und Sericit entstanden. Die Ortoklase haben die Einschlüsse von Plagioklas, Quarz und Sehr wenig Biotit. Der An.-Gehalt der Plagioklase betraegt etwa $20 \%$.

Im Dünnschliff sind die Quarze reichlich vorhanden. In den Quarzen sind wieder Einschlüsse von Feldspat und einige BiotitBlaettchen beobachtet. Ferner stellte ich im Dünnschliff einige Muscovit-Blaettchen fest.

No. 125/52 Augit-Syenitaplit

Das Gestien ist feinkörnig und rötlich gefaerbt. Makroskopisch beobachtete man Feldspat und Gemengteile von dunklen Mineralien.

U. d. Mikroskop sah man, dass das Dünnschliff hauptsaechlich aus Kristaellchen von Feldspat und Augit gebildet sind. Das Gestein ist porphyroid.

Die Ortoklase sind vorherrschend. Sie sind grösser als der anderen Mineralien. Ihre Grösse erreicht $0,24 \times 12 \mathrm{~mm}$. Sie sind 
sehr Einschlussreich (Plagioklas, Magnetit, Apatit, Augtt, einige Hornblende und Sphen).

Die Plagioklase kommen im Dünnschliff meist in Form poly. synthetischer Zwillinge vor. Manche Plagioklase sind stark verwittert. Bei anderen nicht so stark verwitterten Schnitten stellte ich fest, dass die Plagioklase als Andesine vertreten sind.

Die Augite bilden den zweiten Hauptgemengteil des Gesteins. lhre Auslöschungsschiefe ist $42^{\circ}$. Sie sind teilweise uralitisiert.

Ferner stellte ich noch einige Biotit-, Magnetit-, und SphenKristalle fest. Die Biotite sind stark pleochroitisch (leicht gelb bis dunkel braun).

Die Quarze treten in kleinen Kristall-Körnchen sehr zurück.

No. 115/42 Augit-Kersantit

Das Gestein ist dunkel grau gefaerbt. Die dunklen Mineralien sind vorherrschend, die helleren sind dagegen spaerlich.

$U$. d. Mikroskop sind Quer-u. Laengsschnitte von Augit sichtbar. Das Gestein zeigt eine hypidiomorph-körnige Struktur. Manche Augite sind zum Teil uralisiert. Ihre Auslöschungsschiefe betraegt $44^{\circ}$.

Die Plagioklase, die den zweiten Hauptgemengteil des Gesteins bilden, treten im Vergleiche zum Augit sehr zurück. Sie kommen oft polysynthetisch verzwillingten Formen vor. Ihre Auslöschungen sind meist ondulös. Diese Plagioklase haben einen An. Gehalt von ca. $65 \%$. Sie scheinen Labrador zu sein. Als Einschlïsse enthalten sie Augite.

Ausserdem beobachtete ich im Dünnschliff einige Laengs.u. Quer-Schnitte von Horblenden, die einen gelblich grün bis dunkel grünen Pleochroismus zeigen. Ferner stellte jch einige Blaettchen von Biotit und körnchen von Quarz fest.

\section{Literatur}

1 - Tehihatcheff, P. de Asie Mineure, Quatrième partie. Géologie J. Paris, 1807.

2 - Ehaput E. Voyages d'études géologiques et géomorphogéniques en Turquie. Paris, 1936.

3 - Arni, P. Kırşehir, Keskin ve Yerköy havalisindeki arazide vukua gelen zelzele, M. T. A. Ankara, 1938. 
4 - Maucher, A. Das Molybdenglanz und Powellinvorkommen von Hüseyinbeyobas1. Zeitsch. f. Angewandte Mineralogie.

$\overline{5}$ - Baykal. F. Kırıkkale-Kalecik ve Keskin-Bâlâ mintakalarındaki jeolojik etüdler. 1943, M. T. A. raporlarından.

6 - Ladame, G. Le gisement de molybdène de Hüseyinbey.Obası et l'importance économique de ce métal. M.T.A. Sayı : 3, 1937 Ankara.

7 - Kvenko, V. Quelques mines de plomb, de zinc et d'antimoine de la province Nord d'Anatolie (Denek, Akdag, Zara et Turhal). M. T. A. Sayı : 1/37, 1947 Ankara.

(Manuskript eingegangen am 16 November 1955). 